\title{
Les demandeurs de l'aide médicale d'État pris entre productivisme et gestion spécifique
}

Applicants for Medical Assistance State Caught

betweenProductivismandSpecific Management

Los solicitantes de la Ayuda Médica de Estado tomados entre productivismo y

gestión específica

\section{Céline Gabarro}

\section{(Q) OpenEdition}

Journals

Édition électronique

URL : https://journals.openedition.org/remi/5870

DOI : $10.4000 /$ remi. 5870

ISSN : $1777-5418$

Éditeur

Université de Poitiers

Édition imprimée

Date de publication : 1 juin 2012

Pagination : $35-56$

ISBN : 979-10-90426-04-7

ISSN : 0765-0752

\section{Référence électronique}

Céline Gabarro, «Les demandeurs de l'aide médicale d'État pris entre productivisme et gestion spécifique », Revue européenne des migrations internationales [En ligne], vol. 28 - n² | 2012, mis en ligne le 10 octobre 2012, consulté le 14 avril 2022. URL : http://journals.openedition.org/remi/5870 ; DOI : https://doi.org/10.4000/remi.5870 


\section{Les demandeurs de l'aide médicale d'État pris entre productivisme et gestion spécifique}

\section{Céline GABARRO ${ }^{1}$}

T e 27 juillet 1999 a été promulguée la loi créant la couverture maladie univer-

selle (CMU) et son complément l'aide médicale d'État (AME). Ces deux prestations ont été mises en place le $1^{\text {er }}$ janvier 2000 sur l'ensemble du territoire français (métropole et départements d'outre-mer). L'AME offre une couverture maladie aux personnes en situation irrégulière ${ }^{2}$. Son accès est aujourd'hui triplement conditionné : condition de ressources (ne pas dépasser un certain plafond ${ }^{3}$ ), condition de résidence (vivre en France de façon stable et ininterrompue depuis plus de trois mois) et paiement d'un droit d'entrée de trente euros ${ }^{4}$.

L'AME est intégrée à la loi sur la CMU qui vise à ce que l'ensemble des personnes résidant en France soient protégées contre les risques maladie, un des objectifs à terme de la sécurité sociale énoncés dès sa création en 1945. À l'origine, l'affiliation à l'assurance maladie était subordonnée au fait de travailler, les législateurs n'ayant pas pour priorité de traiter la pauvreté, mais plutôt de la prévenir : on assurait le travailleur et sa famille (ses ayants droit) contre des risques plutôt qu'on ne couvrait l'ensemble de la population pour prévenir les conséquences des maladies ${ }^{5}$. Au fil des années, d'autres régimes d'assurance virent le jour, mais ces derniers restaient liés à la notion de travail. Dans les années 1990,

1 Doctorante, allocataire CNAMTS, Université Paris Diderot, Laboratoire URMIS (UMR IRD 205), case courrier 7027,75205 Paris cedex 13 ; celinegabarro@yahoo.fr

2 Les bénéficiaires de l'AME n'ont pas à débourser pour payer leurs soins et médicaments. L'AME prend tout en charge (elle rembourse sur la base d'un $100 \%$ sécurité sociale) sauf si les médecins pratiquent des dépassements d'honoraires ou prescrivent des médicaments non remboursés.

3 Plafond réévalué tous les ans au mois de juillet. En 2012, il est de 648 euros mensuels pour une personne.

4 Ce dernier date de la loi de finances rectificative de 2010.

5 Sur l'histoire de la sécurité sociale, voir notamment les travaux de Robert Castel (1995), Bruno Palier (2005), Numa Murard (2001) et Brigitte Frotiée (2004). 
différents rapports ${ }^{6}$ attestèrent des difficultés que rencontraient les personnes pauvres pour se soigner. Étendre l'assurance maladie à l'ensemble de la population devint alors une priorité, inscrite dans la loi sur la lutte contre l'exclusion et la pauvreté du 29 juillet 1998. Cette extension impliquait toutefois un changement dans le mode d'affiliation (Frotiée, 2004). Lors de son instauration en 2000, la CMU est apparue comme une avancée sociale forte : toute personne résidant en France, y compris les étrangers, à condition qu'ils soient installés en France en situation régulière, pouvait désormais bénéficier d'une couverture maladie de base. Cet accès à l'assurance maladie est cependant moins universel que son nom l'indique. Les étrangers en situation irrégulière en sont exclus pour être dirigés vers l'AME, une offre de soins réduite qui leur est spécifique. L'affiliation à la CMU ou à l'AME se fait ainsi sur critère juridico-administratif : être ou ne pas être en situation régulière. Mais cette distinction n'est pas strictement technique, car dépendre de la CMU ou de l'AME confère des droits différents qui viennent justifier des traitements différenciés.

Depuis sa création, l'AME nourrit les débats politiques et médiatiques. Certains lui sont favorables et la considèrent comme une " avancée sociale " - arguant d'une amélioration de la situation des personnes en situation irrégulière depuis 2000 - et comme un modèle social à sauvegarder en regard de ce qui se passe ailleurs en Europe (Bernard, 2011 ; MIPES, 2003 ; Mbaye, 2009) 7 . Prendre en charge les personnes résidant en France quelle que soit leur nationalité ou leur statut administratif, répond ainsi aux principes énoncés dans le préambule de la Constitution ${ }^{8}$, dans la Déclaration des droits de l'homme et du citoyen et à des soucis humanitaires. Cette vision a néanmoins tendance à faire table rase du passé puisqu'auparavant, les personnes en situation irrégulière bénéficiaient également de soins, mais au même titre et sous le même régime que les Français et les immigrés en situation régulière.

D'autres s'opposent à l'AME, notamment parmi les politiques, dénonçant le coût et les risques d' " appel d'air » ${ }^{9}$ que constituerait un modèle social trop " généreux ». Un argument qui autorise certains parlementaires à réclamer des réformes pour limiter l'accès à l'AME (Carde, 2009). Parmi ces détracteurs, on compte également, même si les raisons en sont bien différentes, ceux qui la jugent régressive et discriminatoire, dans la mesure

6 Le rapport du Centre de recherche d'étude et de documentation « Santé, soins et protection sociale en 1996 » publié en novembre 1997 et l'enquête sur « La progression de la précarité en France et ses effets sur la santé » du Haut Comité de la santé publique en février 1998.

7 Pour des informations complémentaires sur les différents systèmes de santé européens et leur prise en charge des personnes en situation irrégulière, le lecteur peut se référer aux travaux de Médecins du monde (2009), Sandro Cattacin (2007) ou Myriam Hachimi Alaoui (2010).

8 Le Préambule de la Constitution de 1946 expose en son alinéa 11 : «La nation assure à l'individu et à sa famille les conditions nécessaires à leur développement. Elle garantit à tous, notamment à l'enfant, à la mère, au vieux travailleur, la protection de la santé, de la sécurité matérielle, le repos et les loisirs. Tout être qui, en raison de son âge, de son état physique ou mental, de la situation économique, se trouve dans l'incapacité de travailler a le droit d'obtenir de la collectivité des moyens convenables d'existence ».

9 L'idée d'« appel d'air » repose sur une représentation selon laquelle des étrangers, ne participant pas au système de cotisations sociales, viendraient en France uniquement pour se faire soigner (on parle également, souvent à mauvais escient, de « tourisme médical »), ce qui coûterait extrêmement cher au pays. 
où elle réserve un traitement à part aux personnes en situation irrégulière (Fassin, 2002 ; Carde, 2006b ; Maille, 2009 ; Izambert, 2010).

Au-delà de ces débats idéologiques, il convient de s'interroger sur les conséquences que la création de l'AME, sa dissociation de la CMU et les réformes qu'elle a subies depuis 2000 ont sur l'accès aux soins des personnes en situation irrégulière. En nous appuyant sur les données que nous avons recueillies, nous nous intéresserons à trois « champs » dans lesquels la spécificité de l'AME peut avoir des effets concrets en termes d'accès aux soins : le droit et son interprétation d'abord, l'organisation des caisses d'assurance maladie ensuite et enfin les pratiques des agents de ces caisses, que ce soit dans le cadre de la réception des demandeurs ou dans celui de l'instruction des dossiers. Après une description de la méthode d'enquête, nous nous intéresserons en premier lieu aux conséquences de cette distinction juridique entre la $\mathrm{CMU}$ et $\mathrm{l}^{\prime} \mathrm{AME}^{10}$. Nous verrons que cantonner les personnes irrégulières au sein d'une prestation qui leur est propre, favorise un traitement particulier et crée un système de santé à plusieurs vitesses. Nous examinerons ensuite les modalités organisationnelles de cette distinction CMU-AME au sein des caisses d'assurance maladie qui reçoivent les différents publics dans des lieux distincts ou de manières différenciées. Enfin, nous étudierons les pratiques des agents des caisses d'assurance maladie avec d'un côté les agents d'accueil qui reçoivent les demandeurs et de l'autre, les agents du service AME qui instruisent les demandes. Dans ce point, nous serons particulièrement attentive aux effets que peuvent induire les pratiques managériales sous l'emprise d'une gestion productiviste. La combinaison de ces trois angles d'approche donnera ainsi à voir le parcours effectué par les personnes en situation irrégulière et les embûches qu'elles y rencontrent. Nous verrons que ces difficultés peuvent être cumulatives, que le dispositif spécifique de l'AME peut en être la cause, mais aussi que la mise en place de nouvelles normes managériales au sein des caisses d'assurance maladie les accroît.

\section{MÉTHODOLOGIE DE L'ENQUÊTE}

Les dossiers d'AME étant instruits par les caisses primaires d'assurance maladie (CPAM), nous avons observé le fonctionnement de deux d'entre elles, la Caisse des Champs et la Caisse des Lys, qui gèrent chacune l'assurance maladie dans un département comptant une population précaire nombreuse et un taux important de bénéficiaires de la CMU et de l'AME.

À la CPAM des Champs, nous avons mené une enquête principale de près d'un $a^{11}$ dans trois services où, recrutée comme stagiaire, nous avons fait une observation participante : les trois premiers mois, au sein du service de la réglementation CMU-AME qui compte deux agents chargés de retranscrire les réglementations nationales sous forme de notes internes. Elles serviront de référence aux agents du service AME chargés d'ins-

10 Les références à la CMU sont utilisées ici comme point de comparaison pour montrer en quoi l'accueil des bénéficiaires de l'AME et le traitement de leur dossier est spécifique, mais cet article ne traite pas, à proprement parler, de l'accueil des bénéficiaires de la CMU ou de l'instruction de leurs dossiers.

11 Achevée en 2010. 
truire les dossiers et à ceux de l'accueil pour orienter les demandeurs. Nous avons ensuite effectué six mois au service de l'AME situé au siège de la Caisse, à côté du service de la réglementation CMU-AME, qui compte une quinzaine d'agents formés ${ }^{12}$ à l'instruction des dossiers d'AME. Spécialisés dans cette tâche, ils n'instruisent aucun autre type de dossier et ne rencontrent jamais les demandeurs. Nous les appellerons les « gestionnaires de dossiers ». Enfin, en 2010, nous avons réalisé une observation d'un mois et demi dans trois centres d'accueil, sélectionnés pour l'importance des bénéficiaires de l'AME dans leur secteur géographique. Les agents d'accueil de la CPAM des Champs informent les demandeurs de l'AME, vérifient la constitution de leur dossier lors des « entretiens AME », mais n'instruisent pas les dossiers qui sont ici, après vérification, transmis au service de l'AME. Lors de ces observations, nous étions postée derrière les agents d'accueil - que nous appellerons " gestionnaires de clientèle »- et observions ainsi les interactions de guichet et prenions note des commentaires tenus sur leurs actions et réactions.

Une autre enquête a été entreprise auprès de la CPAM des Lys ${ }^{13}$. L'accueil des bénéficiaires de l'AME dans cette caisse s'effectue principalement lors de permanences de l'assurance maladie situées dans les hôpitaux. Notre première approche a consisté à accompagner des demandeurs dans ces permanences. Nous avons ainsi pu rencontrer et échanger avec les demandeurs et partager leurs conditions d'attente. Nous avons ensuite, avec l'autorisation du siège, réalisé trois journées d'observation dans deux permanences hospitalières et dans une troisième située dans une association. Au côté de l'agent, nous « recevions » avec lui les demandeurs sans interférer directement dans l'interaction. Des entretiens informels avec les agents ont eu lieu à cette occasion.

L'observation menée auprès de la CPAM des Lys est moins conséquente que celle effectuée à la Caisse des Champs, mais elle permet de comparer les différences d'organisations et de pratiques observées, et d'appréhender ainsi l'importance de l'organisation sur la façon dont les agents de l'assurance maladie vivent et se représentent leur travail, sur les relations qu'ils entretiennent avec les demandeurs d'AME et sur l'accès aux soins de ces personnes.

\section{LE DISPOSITIF JURIDIQUE PARTICULIER DE L'AME}

\section{3-2003 : les personnes en situation irrégulière mises au ban de l'assurance maladie}

Les personnes en situation irrégulière n'ont pas toujours été exclues du bénéfice de l'assurance maladie. Jusqu'en 1993, les travailleurs en situation irrégulière étaient assurés au régime général au même titre que les autres salariés. Dans ce système d'affiliation par le travail, ils cotisaient, étaient couverts par l'assurance maladie et accédaient aux soins.

12 Comme nous le verrons, la formation des agents est très rapide.

13 Enquête toujours en cours.

REMI 2012 (28) 2 pp. 35-56 
À partir des années 1970 émerge la question des « sans-papiers ». Cette catégorie entre dans la ligne de mire du gouvernement et des médias : obtenir des titres de séjour une fois installé sur le territoire français devient de plus en plus difficile. Peu à peu, les " sans-papiers » font figure de « mauvais » étrangers face aux « bons » étrangers qui seraient ceux en situation régulière (Fassin, 1997). Tandis que les droits des étrangers en situation régulière se développent, ceux des personnes en situation irrégulière diminuent (Siméant, 1998 ; Baron, 2011). La deuxième loi Pasqua du 24 août 1993 relative à la maîtrise de l'immigration et aux conditions d'entrée, d'accueil et de séjour des étrangers en France illustre bien ce climat (Carde, 2009). Subordonnant l'affiliation à l'assurance maladie à la régularité du séjour, cette loi exclut désormais l'ensemble des personnes en situation irrégulière de ce droit. Les travailleurs en situation irrégulière doivent désormais payer de leur poche leurs soins, médicaments et hospitalisations, des dépenses qui peuvent s'avérer très importantes et retarder, voire dissuader, le recours aux dépistages et, au-delà, aux soins curatifs.

Pour les personnes qui ne sont pas affiliées à l'assurance maladie et qui requièrent des soins urgents, il reste le dispositif de l'aide médicale départementale ${ }^{14}$. Subordonné à la seule condition de ressources, il est accessible aux Français et aux étrangers en situation régulière et irrégulière. Avec la création de la CMU, l'ensemble des personnes qui relevaient de ce dispositif sont désormais prises en charge par la CMU, à deux exceptions près : les personnes en situation irrégulière en restent exclues ainsi que les étrangers en situation régulière qui sont en France depuis moins de trois mois. Ces catégories de personnes dépendent alors de l'AME, version réformée de l'aide médicale départementale. Entre 1999 et 2003, l'AME n'est pas encore « la couverture maladie des personnes en situation irrégulière »; ce qu'elle est devenue depuis. Elle est, à ses débuts, une prestation destinée aux étrangers en situation irrégulière, mais également aux étrangers en situation régulière qui, pour bénéficier de la CMU, devaient résider en France depuis plus de trois mois, et doivent au besoin recourir à l'AME durant leurs trois premiers mois de séjour en France. L'AME reste une prestation d'assistance tandis que la CMU dépend de l'assurance maladie. Cette différence assistance/assurance signifie concrètement que les bénéficiaires de l'AME ne sont pas des assurés sociaux contrairement aux bénéficiaires de la CMU. Ils n’ont donc pas les mêmes droits, ni la même reconnaissance.

En $2003^{15}$, présentant l'AME comme un « modèle social » trop favorable, les parlementaires introduisent une condition de stabilité de résidence de trois mois minimum et font sortir l'ensemble des étrangers en situation régulière du public de l'AME. À partir de janvier 2004, l'AME devient la couverture maladie des personnes en situation irrégulière, une prestation potentiellement stigmatisante.

14 Dispositif d'aide sociale, anciennement appelé assistance médicale gratuite, a été créé par la loi du 15 juillet 1893. Voir à ce sujet Carde (2006a) et Frotiée (2004).

15 Loi de finances rectificative pour 2003 votée le 30 décembre 2003. 


\section{Aide Médicale d'État ou Couverture Maladie Universelle : les conséquences juridiques d'une distinction légale}

Avec l'AME, les parlementaires instaurent une distinction légale entre, d'un côté, les personnes françaises ou étrangères en situation régulière et, de l'autre, les étrangers en situation irrégulière. Une discrimination légale qui pointe du doigt les personnes en situation irrégulière et leur réserve un traitement spécifique. La délivrance de l'AME est désormais subordonnée à un plus grand nombre de conditions et remboursera moins de soins que la CMU, une différence de qualité de prise en charge qui ne cessera de s'amplifier au fur et à mesure des réformes du dispositif (Carde, 2009).

Dès 2000, la CMU et l'AME sont soumises à une condition de ressources. Cette condition, cependant, ne s'applique pas de la même manière dans les deux cas. Pour l'AME, c'est l'ensemble de la couverture qui y est soumis. Si les ressources du demandeur dépassent le plafond fixé, il ne pourra pas bénéficier de cette couverture maladie. Seules ses hospitalisations, pour des soins considérés comme urgents et vitaux, seront prises en charge ${ }^{16}$. La CMU comprend, elle, deux prestations, la CMU de base qui est une couverture maladie correspondant à la part " sécurité sociale » et la CMU complémentaire (CMUC) qui est une mutuelle. Or la condition de ressources ne s'applique qu'à l'octroi de la CMUC. Subséquemment, une personne en situation régulière dont les ressources mensuelles sont supérieures au plafond fixé, bénéficiera de la CMU de base ${ }^{17}$ pour les dépenses prises en charge au titre de la sécurité sociale (comme ce serait le cas pour un travailleur sans mutuelle), mais ne pourra prétendre à la CMUC : le ticket modérateur ${ }^{18}$ restera à sa charge. Ainsi, à revenu égal, les personnes en situation régulière et celles en situation irrégulière seront traitées distinctement du simple fait de leurs différents statuts de résidence.

Le bénéfice de la CMUC n'ouvre pas non plus le droit aux mêmes prestations que l'AME. La CMUC comprend un panier de soins plus important que l'AME. Par exemple, le ticket modérateur des prothèses dentaires ou optiques, remboursé par la CMUC, ne l'est pas par l'AME qui se limite à appliquer le taux du forfait de base de la sécurité sociale. Ainsi, la base forfaitaire pour les lunettes étant extrêmement faible, le bénéficiaire de l'AME doit assumer la quasi-totalité du coût de la monture et des verres, des frais trop importants pour des personnes vivant avec moins de 648 euros par mois (plafond fixé pour l'AME en 2012). Le problème est le même pour les soins et prothèses dentaires.

16 Le fonds des soins urgents et vitaux (FSUV) ne prend en charge que les soins urgents et vitaux tels que définis à l'article L254-1 du Code de l'action sociale et des familles (CASF) et par la circulaire DHOS/DSS/DGAS n ${ }^{\circ} 141$ du 16 mars 2005. Il permet, pour certaines opérations, de rembourser aux hôpitaux les frais engagés pour des personnes en situation irrégulière qui ne bénéficient pas de l'AME. Découragées par leurs administrations d'engager des impayés, les équipes médicales peuvent refuser d'entreprendre des opérations considérées comme « non vitales ».

17 L'accès exonéré de cotisations à la CMU de base est conditionné par les ressources. Pour la période du $1^{\text {er }}$ octobre 2011 au 30 septembre 2012, le plafond est fixé à 9164 euros annuels. En cas de ressources supérieures, l'assuré doit cotiser à hauteur de $8 \%$ de ses revenus.

18 Part des dépenses de santé qui reste à la charge de l'assuré après remboursement de l'assurance maladie. 
Distinguer l'AME de la CMU a également permis, au fil des années, de mettre en place des réformes propres à l'AME dont l'accès a ainsi été maintes fois durci sans que soit pour autant modifié celui de la CMU. Ainsi, l'accès à l'AME était gratuit jusqu'à ce que la loi de finances rectificative de 2010 instaure un droit d'entrée annuel de trente euros par personne majeure ; une disposition particulièrement préjudiciable pour une population ayant de très faibles ressources. A contrario, aucun droit d'entrée n'a été instauré pour la CMU. En dissociant l'AME de la CMU, les parlementaires ont rendu possible une limitation de l'accès à l'AME, tout en préservant la CMU. Par ailleurs, l'organisation en deux régimes distincts ne s'arrête pas à un traitement différent des étrangers en situation irrégulière en regard des étrangers en situation régulière et, a fortiori, des Français. Elle rend aussi plus vulnérables tous les étrangers, quelle que soit leur situation de résidence, car, contrairement aux Français, tout étranger risque de basculer dans la catégorie des irréguliers (Ferré, 1997 ; Fassin, 2001) comme ce fut le cas des ressortissants communautaires inactifs en 2007 (Math, 2009 ; Gabarro, à paraître).

Du point de vue des agents de l'assurance maladie, cette différence de traitement dans l'accès aux soins se justifie par la différence de couverture maladie. Ils considèrent que ce n'est pas parce que les étrangers sont en situation irrégulière qu'ils sont traités différemment, mais parce qu'ils dépendent de l'AME. Et c'est parce qu'ils sont bénéficiaires de l'AME qu'ils ont une prise en charge inférieure, une offre de soins plus réduite. Se référer à cette division légale entre la CMU et l'AME permet aux agents de dépolitiser et déshumaniser le problème : ils ne traitent pas des personnes en situation irrégulière ou régulière, mais des bénéficiaires de l'AME et des bénéficiaires de la CMU.

\section{LES EFFETS DES MODALITÉS ORGANISATIONNELLES : POUR LA CMU LES CENTRES D'ASSURANCE MALADIE, POUR L'AME DES GUICHETS SPÉCIFIQUES}

Une des premières conséquences pratiques de la séparation légale de la CMU et de l'AME concerne l'organisation de l'accueil des demandeurs de l'AME. Bien que de nombreuses associations aient plaidé pour la mise en place d'un guichet unique ${ }^{19}$, les CPAM des Champs et des Lys ont en commun de réserver un accueil « à part », voire " à l'écart », pour les demandeurs de l'AME, contrairement aux autres assurés sociaux, les demandeurs de la CMU y compris.

En ce qui concerne l'application des instructions (conditions d'obtention, application de nouvelles réformes), les CPAM - organismes de droit privé exerçant une mission de service public - sont sous l'autorité hiérarchique de la Caisse nationale d'assurance maladie des travailleurs salariés (CNAMTS). Mais sur le plan organisationnel, elles fonctionnent comme des organismes autonomes : chacune gère ses employés, ses locaux et son organisation. Partant, chaque CPAM décide librement du nombre de centres qui seront implantés sur son territoire, de leurs spécificités, des horaires d'ouverture, de leur taille

19 C'est-à-dire un guichet où toutes les personnes seraient reçues quel que soit leur statut ou leur demande. Cela permettrait de limiter les situations discriminatoires, mais aussi d'éviter des « descentes » de police ciblées et un repérage trop évident des personnes en situation irrégulière. 
ou encore du type d'usagers reçus. Toutefois, ces variantes départementales n'ont pas d'incidence sur l'accueil des assurés sociaux (du régime de droit commun et de la CMU). Ils se rendront dans un centre d'assurance maladie de la CPAM et seront reçus de la même manière, qu'il s'agisse d'un centre de quartier, d'un point d'accueil ou d'un " pôle d'activité $\gg{ }^{20}$. En revanche, l'accueil des demandeurs de l'AME diffère beaucoup selon les départements. Ces variations sont difficiles à comprendre pour les bénéficiaires de l'AME qui changent de département et se trouvent confrontés à une nouvelle organisation. Il leur faut alors saisir le fonctionnement, bien souvent obscur à première vue, de leur nouvelle CPAM, sous peine de voir leur accès aux soins retardé, voire refusé. Or il s'avère que c'est surtout sur l'accueil du public de l'AME que les directions des CPAM semblent jouer pour ajuster leur gestion des flux d'usagers en attente et la répartition de leur charge de travail. Et c'est ainsi, par l'accueil qui leur est réservé (fortement stigmatisant dans certains départements), que les demandeurs et bénéficiaires de l'AME apprennent leur statut d'usagers de « seconde classe ».

À la CPAM des Lys, il existe trois types d'accueil : des centres d'accueil de l'assurance maladie standard $(n=17)$ qui sont réservés aux assurés sociaux, des centres dits " de grande précarité » $(\mathrm{n}=2)$ exclusivement réservés aux demandeurs de la CMU et de l'AME et enfin, les accueils extérieurs (des permanences de l'assurance maladie situées dans les hôpitaux ${ }^{21}$ et les associations). Les demandeurs de l'AME peuvent s'informer dans les centres d'accueil standard (même s'ils y sont rarement orientés), mais les agents de ces centres n'étant pas formés à l'AME, ils ne peuvent pas vraiment les renseigner et ne font que leur fournir le formulaire et les rediriger dans la voie qui leur est réservée.

Dans les deux centres de grande précarité, les dossiers de CMU et d'AME sont instruits en totalité sur place, jusqu'à la délivrance de l'attestation. Du coup, les demandeurs peuvent attendre plusieurs heures avant d'être reçus. Pour s'assurer d'être accueillis dans les meilleurs délais, des demandeurs font la queue dès $6 \mathrm{~h}$ du matin à l'extérieur des centres, attendant leur ouverture.

La situation est pire encore dans les permanences hospitalières où les demandeurs de l'AME se rendent majoritairement puisqu'ils y sont (quasi) systématiquement adressés par la plateforme téléphonique, les assistantes sociales et les médecins, surtout en cours ou en sortie d'hospitalisation - la plupart des demandeurs n'entamant les démarches qu'une fois le recours aux soins est devenu absolument nécessaire. Une fois familiarisés avec ces

20 Selon les départements, les «centres d'accueil » n'ont pas forcément la même dénomination. Certaines CPAM ont divisé leur accueil en « pôles d'activité » : il y a des centres qui ne reçoivent que les salariés et d'autres uniquement les professionnels de santé. D'autres CPAM parlent de « centres ». Enfin au sein d'une même CPAM il peut exister différents types de centres : les « petits » qui n'assurent qu'une mission d'accueil et sont ouverts seulement quelques jours dans la semaine et les " gros » centres qui comprennent également des services de « back office » où les agents instruisent les dossiers.

21 Nous utiliserons le terme " permanence hospitalière ». Ces permanences sont à distinguer des PASS (Permanences d'Accès aux Soins de Santé, dispositif visant à assurer l'accès aux soins et à la couverture maladie des personnes démunies en mettant en réseau les professionnels de la santé et du social) : l'assurance maladie assure ici une permanence extérieure : un bureau de l'hôpital est attribué à un agent de l'assurance maladie qui s'occupe des ouvertures de droits à l'assurance maladie des patients de l'hôpital et des demandeurs de l'AME.

REMI 2012 (28) 2 pp. $35-56$ 
permanences, les bénéficiaires de l'AME y retournent pour leur demande de renouvellement et l'indiquent également à leurs amis malades. Les permanences hospitalières sont ainsi en quelque sorte étiquetées « permanences AME $»^{22}$. Ces locaux sont toutefois mal signalés et difficiles à trouver. Dans un des hôpitaux où nous avons conduit nos observations, le bureau était situé à l'étage sans autre signalétique qu'une feuille collée sur un des murs de l'escalier indiquant les horaires de la permanence. Les demandeurs devaient faire la queue en bas de celui-ci, seul endroit équipé d'un banc. Ces permanences hospitalières sont au nombre de dix. En 2011, vingt agents y travaillaient ${ }^{23}$, contre quarante en 2000. Les départs en retraite n'ont pas été remplacés alors que le nombre de bénéficiaires de l'AME a été multiplié par sept. La réduction du coût de la gestion de l'accueil est devenue prioritaire pour toutes les caisses d'assurance maladie. Pour limiter les coûts locatifs et les frais de personnel, les Caisses des Champs et des Lys ferment actuellement les centres dont les murs ne leur appartenaient pas, regroupant leurs accueils et mutualisant leurs effectifs. Les agents des permanences hospitalières ne sont pas suffisamment nombreux pour gérer le flux des demandeurs, d'autant plus qu'ils doivent également traiter les demandes des assistantes sociales de l'hôpital pour les patients hospitalisés. Dans de telles conditions, ils s'organisent tant bien que mal, recevant, par exemple, les demandeurs de l'AME le matin et les assistantes sociales l'après-midi. Mais l'organisation varie toutes les semaines en fonction du volume des demandes des assistantes sociales et des réunions fixées par la CPAM. Les horaires d'ouverture sont donc affichés au guichet chaque lundi. Le nombre de demandeurs d'AME accueillis varie ainsi en fonction des agendas des agents et de leur manière de travailler. Ils peuvent recevoir vingt-cinq personnes une journée et douze la suivante. L'agent de permanence arrive à $8 \mathrm{~h} 30$ et distribue des tickets aux personnes présentes. Certains essaient d'en distribuer le maximum pour accueillir le plus de personnes possible, d'autres préfèrent en distribuer moins, mais prendre plus de temps pour les dossiers. Une fois atteint le quantum de tickets à distribuer, les autres personnes sont invitées à partir.

Confrontés aux aléas de cette organisation en " flux tendu », les demandeurs refoulés plusieurs fois viennent de plus en plus tôt pour accroître leur chance d'être reçus ; d'autant que si leur dossier n'est pas complet, il faudra revenir un autre matin. Ici, la gestion spécifique des demandeurs de l'AME se traduit par un traitement assez dégradant de ces personnes malades qui se retrouvent à faire la queue devant la permanence dès $3 \mathrm{~h}$ du matin, voire, pour celles qui n'ont aucun moyen de transport, dès la veille au soir, obligées de dormir sur place. L'hôpital étant fermé la nuit, les agents de la Sécurité les envoient aux Urgences, qui les renvoient à leur tour. Ballotés d'un endroit à l'autre, ils ne sont autorisés à retourner dans la partie administrative de l'hôpital qu'à son ouverture. Beaucoup d'hôpitaux n'ayant pas de salle d'attente adéquate, les demandeurs patientent dans les couloirs ou parfois sur des bancs en plein milieu des halls à la vue de tous, "pas celui en bois de l'autre côté du hall, le banc en pierre, froid» expliquait l'un d'eux, précisant que s'ils s'asseyaient sur le banc en bois, on les rappelait à l'ordre : «leur place n'était pas ici ». Ils se sentent traités « comme du bétail » et considérés avec

22 D'ailleurs lorsque nous demandons au personnel de l'accueil central des hôpitaux où se situe la permanence de la sécurité sociale, il est courant de les entendre rétorquer comme une évidence : «L'aide médicale d'État? ». Lors de nos observations, nous avons pu noter que les panneaux sur la porte des permanences hospitalières indiquent clairement « $\mathrm{AME}$ ».

23 Ces mêmes agents assurent également les permanences dans les associations. 
mépris par le personnel hospitalier qui les regarde comme s'ils leur «prenaient quelque chose ». L'inadaptation des lieux à la réception de longues files d'attente (comme le faisait remarquer un agent d'accueil nous indiquant la permanence : " il y a plein de monde qui fait la queue, vous ne pouvez pas la rater ») contraint les demandeurs à s'auto-organiser pour faire respecter leur ordre d'arrivée : le premier sur place établit une liste de passage sur une feuille de papier, obtenue à l'accueil, où chacun inscrit son nom dès son arrivée. Parfois, des demandeurs inscrivent un ami qui ne viendra que plus tard, ou certains décident de partir et proposent leur place à une autre personne, ce qui peut provoquer certaines frictions. Quand le guichet ouvre, la liste est remise à l'agent pour la distribution des tickets. Dans les salles d'attente étriquées, la tension monte sensiblement au moment de la distribution. Ceux qui comprennent qu'ils ne seront pas reçus dans la journée tentent au moins de s'assurer, auprès de l'agent, que leur dossier est complet. Bien souvent l'agent refuse de répondre, estimant que les questions risquent de se multiplier et que ce temps accordé aux réponses l'empêchera de recevoir un maximum de personnes.

Comparée à cette situation, la gestion de l'accueil à la CPAM des Champs paraît plus simple et moins humiliante pour les étrangers en situation irrégulière. Mais, si les conditions d'attente aux guichets diffèrent, nous allons voir que d'autres éléments interfèrent sur le travail des agents administratifs - qu'ils soient gestionnaires de clientèle ou gestionnaires de dossiers -, influant sur la qualité de l'accueil et de l'instruction des dossiers et constituant d'autres types d'embûches.

\section{DES NORMES MANAGÉRIALES AUX PRATIQUES DES AGENTS DES CAISSES}

\section{À l'accueil : des impératifs managériaux qui rendent plus difficile la réception des bénéficiaires de l'AME}

Jusqu'en 2004, la CPAM des Champs recevait les demandeurs et bénéficiaires de l'AME dans un seul centre pour l'ensemble du département. Les personnes devaient parfois se déplacer d'un bout à l'autre du territoire pour obtenir un renseignement. Ce système a cessé à la suite de plaintes des associations qui soulignaient que ce type d'accueil, réservé à des personnes en situation irrégulière, facilitait les rafles policières. Depuis, les demandeurs de l'AME sont reçus dans les mêmes centres d'accueil que les assurés sociaux. Toutefois, leur réception répond à des procédures particulières.

À la CPAM des Champs, il y a trois stades dans l'accueil : le guichet du «préaccueil », le « deuxième niveau », puis le troisième stade (les « rendez-vous » et les « entretiens AME ») (Martin, 2011). Les deuxième et troisième stades ont lieu dans un box. Toutes les personnes qui se rendent dans les centres passent par le pré-accueil pour indiquer l'objet de leur visite. Â partir de là, deux circuits se dessinent en fonction du statut du demandeur. Pour les assurés sociaux (régime général ou CMU), soit le gestionnaire de clientèle peut répondre à leur requête dès le pré-accueil et les personnes repartent informées, soit leur demande est plus longue à traiter et elles sont orientées vers le « deuxième niveau », dans 
un box. Si l'agent de « deuxième niveau » n'a pas réussi à régler le problème, il donne à l'assuré un « rendez-vous ».

Les demandeurs de l'AME relèvent quant à eux d'un autre circuit. Eux aussi commencent par le pré-accueil où les gestionnaires de clientèle leur délivrent un dossier et les informent sur les justificatifs à apporter. Mais, s'ils souhaitent être aidés dans la constitution de leur dossier, ils sont envoyés directement au troisième stade de l'accueil en « entretien AME », sans passer par le deuxième stade. Or, si le deuxième niveau est assuré immédiatement, " au fil de l'eau », les entretiens AME se font uniquement sur rendezvous, contraignant les demandeurs de l'AME à attendre un certain temps avant de pouvoir faire vérifier leur dossier.

La multiplication des degrés d'accueil s'explique par des impératifs managériaux. Dès son arrivée en centre d'accueil, les temps d'attente et de réception de l'usager sont chronométrés. Pour être reçu, ne serait-ce qu'au guichet, il doit prendre un ticket de passage. Sa délivrance est enregistrée et permet d'établir des statistiques. Ces temps chronométrés permettent à la CPAM d'obtenir et de conserver sa norme qualité ${ }^{24}$, d'évaluer ses différents centres d'accueil et d'accorder des primes et des promotions à leurs salariés. Les agents doivent respecter les temps d'attente et de réception propres à chaque catégorie d'accueil. Le temps de réception ne doit ainsi pas dépasser trois minutes au pré-accueil, vingt minutes en deuxième niveau, trente minutes en rendez-vous et en entretien AME. Tenir ces temps est censé limiter le délai d'attente moyen des usagers à une vingtaine de minutes maximum, norme d'un service « de qualité ».

Les « entretiens AME » ont été instaurés par la Caisse nationale. Ils permettent aux gestionnaires de clientèle de s'assurer que le dossier est bien complet et de mieux contrôler les déclarations de ressources des demandeurs. Ces entretiens répondent à une logique managériale : si ce travail est bien fait, le service AME, à qui sont transmis ces dossiers pour instruction, ne recevra que des dossiers complets qu'il pourra traiter rapidement. Ils visent aussi à lutter contre la fraude en permettant de comparer le montant des ressources déclarées aux sommes dépensées. Lors de l'entretien, les gestionnaires de clientèle interrogent les demandeurs sur la somme qu'ils consacrent mensuellement à leur logement, leur nourriture, leurs vêtements, leur maquillage, leurs charges ou encore leurs sorties. Si le montant des dépenses (qui sont évaluées très grossièrement par des demandeurs ne s'attendant pas à cet exercice) dépasse celui des ressources, ne serait-ce que de quelques euros, les agents leur demandent de revoir leurs déclarations.

Normalement, selon les instructions internes à la CPAM des Champs, les entretiens AME devraient être effectués dès que les demandeurs se présentent au pré-accueil. Cela supposerait soit d'affecter un gestionnaire clientèle à ces questions, soit de demander à l'agent responsable du deuxième niveau de les accueillir au même titre que les autres

24 Les caisses sont en effet soumises à des normes qualités, certifiées « norme ISO » (International Standard Organisation), dans chacune de leurs activités, qui leur permettent de s'assurer une bonne place dans le classement national et conditionnent l'obtention de primes pour leur direction. Cette norme est décrite, sur le site de l'assurance maladie (www.ameli.fr) comme une « reconnaissance, par un organisme extérieur, du travail accompli par l'ensemble du personnel afin de satisfaire nos clients et d'améliorer le service rendu». 
usagers, mais en trente minutes au lieu de vingt. Cependant, recevoir des personnes en trente minutes augmenterait, ipso facto, le délai d'attente moyen, nuisant aux objectifs « qualité » des agents et des centres. C'est pourquoi deux des trois centres observés reçoivent uniquement les demandeurs de l'AME sur rendez-vous, quitte à les faire revenir et à augmenter d'autant le délai d'instruction de leur dossier.

Ces impératifs de productivité se combinent aussi avec des problèmes d'effectifs. Pour assurer à la fois le pré-accueil, le deuxième niveau, les rendez-vous et les entretiens AME, chaque centre doit être doté au minimum de cinq agents (sans compter le travail de « back office » qui leur est demandé) ${ }^{25}$. Mais les centres sont en général en sous-effectif du fait d'une politique d'embauche qui ne prévoit pas le remplacement des départs. En conséquence, les entretiens AME sur rendez-vous sont privilégiés et le nombre de jours par semaine qui leur sont réservés varie selon les centres et la disponibilité de leurs agents. Dans certains centres, les demandes d'AME étant importantes, il faut parfois attendre un mois pour obtenir un rendez-vous. En cas de manque d'effectifs, c'est la première mission à être reléguée, ces demandes n'étant pas considérées prioritaires. Pour une personne malade, cela peut signifier un mois d'attente pour constituer son dossier, suivi de deux mois pour que celui-ci soit instruit, soit la limite du délai légal ${ }^{26}$. Il s'agit donc d'un retard aux soins conséquent qui conduit certains demandeurs à envoyer leur dossier par la poste au service AME, au risque de le voir retourné ou refusé, parce que mal rempli.

La pression managériale à laquelle sont soumis les agents chargés de l'accueil affecte leurs rapports aux bénéficiaires. Pour tenir les temps d'attente et de réception, par lesquels ils sont évalués, les agents doivent sacrifier les considérations de politesse ou d'aide sociale. Peu parmi eux prennent le temps d'expliquer complètement les démarches aux personnes qui les sollicitent. Au pré-accueil, la plupart des agents indiquent rapidement la liste des documents à apporter sans s'informer de la situation des requérants. Ce n'est qu'au cours du rendez-vous, le mois suivant, qu'ils obtiennent les informations nécessaires et s'aperçoivent, bien souvent, qu'il leur manque un document qu'ils auraient pu apporter. Pour cela, ils devront attendre un autre rendez-vous, un mois plus tard. Les tensions aux guichets se cristallisent particulièrement quand les demandeurs ne comprennent pas bien le français et/ou n'arrivent pas à se faire comprendre. La plupart des gestionnaires de clientèle, soucieux de ne pas affaiblir leur rendement, leur demandent alors de revenir accompagnés, ce qui rallonge encore les délais d'obtention de l'AME et, par là même, leur accès aux soins.

$25 \mathrm{Il}$ faut en général au minimum deux agents au pré-accueil pour assurer un temps d'attente faible. On parle de « front office » pour le travail de réception au guichet (pré-accueil, deuxième niveau, entretiens AME) et de « back office » pour le travail effectué « à l'arrière », soit derrière le guichet, dans un espace collectif invisible des usagers. Les caisses étant en sous-effectif, il arrive fréquemment que des agents d'accueil soient appelés en renfort pour « solder le stock » de dossiers en attente et éviter à la Caisse d'accorder des droits « à des personnes qui ne devraient pas en bénéficier ». En cas de gros retards, il arrive également que les centres d'accueil soient fermés une journée par semaine pour que l'ensemble des agents traite les dossiers en attente.

$26 \mathrm{Si}$ la norme qualité fixe le délai de traitement d'un dossier AME à un mois, le délai légal est de deux mois. À plusieurs reprises, durant notre enquête, la CPAM des Champs a dépassé ce délai, le nombre de dossiers à traiter excédant la capacité de ses effectifs.

REMI 2012 (28) 2 pp. 35-56 


\section{L'impact des réformes de l'AME sur le travail des agents des caisses}

La gestion productiviste de l'accueil des publics et de la prise en charge des dossiers par les services des CPAM a de forts effets sur le travail quotidien des agents de l'assurance maladie, d'autant plus qu'à prestation spécifique, procédures spécifiques. L'AME étant différente de la CMU, les demandeurs n'ont pas à répondre aux mêmes conditions, à remplir les mêmes formulaires ou à fournir les mêmes pièces pour obtenir l'une ou l'autre. L'existence de deux prestations distinctes multiplie ainsi les procédures, les services, les structures et accroît le travail des gestionnaires de clientèle.

Ces derniers sont en première ligne et doivent pouvoir informer tout demandeur sur n'importe quelle question : régime général, CMU, AME, arrêt maladie, maternité, accident du travail, etc. Lors de leur formation, ils acquièrent une somme de connaissances techniques et juridiques, mais très peu concernant l'AME. En 2010, à la CPAM des Champs, la formation au métier de gestionnaire de clientèle est de deux mois, comprenant à la fois des cours et des temps d'immersion sur le terrain. Une seule journée est consacrée à la présentation de la CMU et de l'AME. L'essentiel de la formation porte sur l'apprentissage des différents régimes de droit commun - il en existe une centaine - et des prestations auxquelles ils donnent accès. Les connaissances à acquérir sont très denses, d'autant que les règles de droit ne cessent d'évoluer et les modalités de remboursement d'être réformées. En théorie, ces agents se doivent d'être au fait de ces modifications, mais, en pratique, ils les maîtrisent difficilement. Dans le cours de leur travail, ils sont informés des réformes par les notes d'instruction qu'ils reçoivent par Intranet comme l'explique l'un d'eux : "La législation de l'assurance maladie représente 18000 textes de loi en mouvement perpétuel. Il est difficile de tous les connaître parfaitement. D'être au courant des modifications. C'est pour ça qu'il y a des notes d'instruction. On est censé les connaître, mais moi je préfère les imprimer, pour certaines, pour les avoir sous le coude et pouvoir m'y référer en cas de besoin plutôt que d'avoir à rechercher sur l'Intranet. En pré-accueil, je n'ai pas le temps de me connecter et de rechercher la note ». Les cadres de l'accueil impriment les notes d'instruction et demandent aux employés de les parapher pour s'assurer qu'ils les ont bien lues. Cependant, l'accueil étant ouvert toute la journée, les gestionnaires de clientèle ne quittent jamais le guichet et ont à peine le temps de lire ces notes. De même, il est difficile de les réunir tous pour des réunions d'information. Les cadres ne vérifient finalement ni la lecture ni la bonne compréhension de ces notes, ni même, parfois, la mise en pratique des informations qu'elles contiennent.

De plus, la loi évoluant, les réformes, surtout en matière d'AME, sont incessantes et les agents des caisses s'y perdent. Il arrive qu'ils ne sachent plus quel droit s'applique : ce fut le cas avec les ressortissants communautaires inactifs pour qui la règle n'a cessé d'être modifiée pendant un an (Gabarro, à paraître). L'organisation de la CPAM des Champs accentue encore cet effet : les gestionnaires de clientèle n'instruisant pas ces dossiers, l'AME n'est pas une priorité pour eux. Ils prêtent ainsi moins attention aux notes d'instruction AME, qu'ils lisent généralement « en diagonale » entre deux assurés, et en accordent plus à celles qui concernent les actions qu'ils effectuent le plus souvent et leur semblent les plus utiles. Les réformes de l'AME leur paraissent d'autant plus éloignées de leurs préoccupations que ces notes sont rédigées par le service de réglementation CMU-AME, dirigé par les cadres du service AME. Ce service s'intéresse ainsi plus aux 
procédures d'instruction des dossiers qu'aux questions propres à l'accueil. Ne sachant comment sont instruits les dossiers d'AME, les gestionnaires de clientèle ne comprennent donc pas toujours ce qui se joue dans ces nouveautés, mais n'appellent pas pour autant le service AME, soit qu'ils n'en aient pas le temps, soit qu'ils aient peur d'y être mal reçus. Ils interprètent alors comme ils le peuvent les notes d'instruction et introduisent parfois des pratiques de droit commun qui ne devraient pas s'appliquer. Ainsi certains réclament aux demandeurs un acte de naissance ou de mariage, alors que l'obtention de l'AME repose sur des déclarations sur l'honneur. L'inscription du nom de l'époux dans le dossier fait foi et aucun acte de mariage ou livret de famille n'a à être réclamé. De même, les bénéficiaires de l'AME relevant d'un régime « à part », ils n'ont pas besoin de joindre un acte de naissance à leur demande, un passeport suffit.

Cette confusion des règles et ce manque de formation alimentent des erreurs de droit et donnent lieu à des pratiques variables selon les gestionnaires de clientèle. Les " mauvaises » pratiques peuvent même devenir la règle à l'échelle d'un centre car, quand ils ne savent pas, les agents ont surtout tendance à imiter leurs collègues plutôt que d'appeler le service AME ou demander un renseignement à leur cadre. Avouer que l'on n'a pas compris une règle de base peut être mal vu. En outre, le cadre, qui n'est pas forcément, lui non plus, très au fait de l'AME, peut se fier à ses agents. En effet, dans deux des centres où nous avons enquêté, les cadres de l'accueil estimaient ne pas être en capacité de répondre aux questions concernant l'AME car ils n'avaient jamais travaillé à l'accueil, ou alors « au temps où l'AME n'existait pas ». Certaines erreurs d'application sont monnaie courante dans les centres où, par exemple, la quasi-totalité des gestionnaires de clientèle observés demandaient des justificatifs de résidence de plus de trois mois et de moins d'un an aux demandeurs venant renouveler leur AME. Une exigence qui ne s'applique pourtant qu'à la première demande, puisque pour les renouvellements, une preuve de moins de douze mois suffit.

Ce type de problèmes s'observe moins en matière de CMU car, en plus de leur mission d'accueil, les gestionnaires de clientèle instruisent les demandes de CMU en « back office ». Ils connaissent ainsi bien mieux la législation de la CMU, la finalité des documents requis et sont mieux à même d'informer les demandeurs. Ils vont également prêter une plus grande attention aux notes d'instruction relatives aux modifications des conditions d'obtention ou d'application de la CMU. Le savoir théorique et pratique accumulé par expérience rend d'autant plus aisé et rapide la compréhension des notes, de leurs tenants et aboutissants. Enfin, ils côtoient quotidiennement les agents spécialisés dans l'instruction des dossiers de CMU, qui travaillent dans les mêmes locaux qu'eux, alors qu'ils ne connaissent pas les employés du service AME, situé au siège de la CPAM. Les gestionnaires de clientèle obtiennent ainsi des informations sur les réformes de la CMU ou l'avancée du traitement des dossiers au détour d'un déjeuner ou d'une pausecafé. Une communication qui leur est impossible avec les agents du service AME à qui, pourtant, ils transmettent les dossiers pour instruction. Au final, le traitement des dossiers d'AME et l'accès aux soins pour les personnes en situation irrégulière dépendent très fortement de l'organisation des caisses et de la gestion de leurs services dédiés à cette couverture maladie. 


\section{Des impératifs de production et une politique du chiffre qui favorisent le « retour » des dossiers}

Les gestionnaires de clientèle sont donc évalués en fonction de leur capacité à tenir les temps d'attente et de réception. Mais ils le sont également selon leur taux d'erreurs, calculé au prorata du nombre de dossiers qu'ils ont envoyés au service AME et du nombre que ce dernier leur a retourné pour manque de justificatifs (dossiers incomplets). Ces « erreurs » sont comptabilisées par les cadres de l'accueil. Lors des évaluations annuelles, elles sont reprochées aux agents et prises en compte pour juger des promotions ou augmentations. L'enjeu pour les gestionnaires de clientèle est donc élevé et, ne comprenant pas toujours leurs erreurs ${ }^{27}$, ceux-ci vont avoir tendance à exiger du demandeur la production d'un maximum de documents qui ne conditionnent pourtant pas l'obtention de l'AME : multiplication des justificatifs de résidence en France avec l'espoir que l'un d'entre eux sera accepté par le service AME ; documents non requis tels qu'un justificatif de domicile ou l'original du passeport avec lequel ils sont entrés en France pour prouver leur ancienneté de résidence, etc. Bref, plutôt que de courir le risque d'envoyer un mauvais dossier et d'être sanctionnés, les gestionnaires de clientèle préfèrent faire revenir le demandeur avec de «nouvelles preuves ». Ces exigences supplémentaires sont liées à la procédure d'apprentissage du droit de l'AME par tâtonnement ou essais-erreurs ${ }^{28}$ et elles accroissent les difficultés pour les personnes en situation irrégulière alors même qu'elles remplissent toutes les conditions requises. Obligées de reprendre rendez-vous, bien souvent un mois plus tard, pour apporter de nouveaux éléments, souvent inutiles, elles voient leur accès aux soins retardé alors que, dans bien des cas, si elles avaient directement envoyé leur dossier au service AME, elles auraient obtenu un accord et reçu leur attestation sans problème.

Anticiper les retours de dossiers d'AME incomplets peut cependant parfois faire gagner du temps aux demandeurs et éviter un surcroît de travail aux gestionnaires de clientèle puisqu'un dossier retourné suit exactement le même circuit qu'un premier envoi. Au vu de l'ensemble des délais entre l'entretien AME et l'obtention de celle-ci, un dossier, qui parce qu'incomplet au premier passage fait deux fois le circuit, prend au total six mois.

Les gestionnaires de dossiers du service AME sont, quant à eux, également soumis à des normes de productivité qui favorisent aussi les renvois de dossiers (les « retours »). Ces agents, qui ne traitent que des dossiers d'AME (de tout type : première demande, renouvellement, soins urgents et vitaux), vérifient chaque dossier qui, dès lors qu'il est passé par leurs mains, suit une des trois directions possibles. S'il est considéré comme complet, il passe dans la case des « accords ». Si la demande est considérée infondée en droit, il atterrit dans la case des « refus ». Enfin, s'il manque des pièces justificatives, il est mis dans la case des « retours » (le dossier est renvoyé au demandeur pour complément). Or, chaque gestionnaire de dossiers est évalué en fonction du nombre de dossiers qu'il instruit et de son taux d'erreur. Pour qu'un dossier mis dans la case « accords »

27 Elles sont notées dans la fiche qui accompagne le dossier comme par exemple «pas de preuve de résidence ». Le service AME n'explique toutefois pas en quoi le document envoyé ne vaut pas comme preuve.

28 Les agents d'accueil sont ainsi formés «par l'erreur » par le service AME : c'est uniquement lorsqu'ils se trompent qu'ils sont repris par ces agents. 
soit définitivement accordé, il doit être revérifié par un contrôleur délégataire de l'Agent comptable. Si une erreur a été commise, cette dernière est consignée et le dossier repris par le gestionnaire de dossiers avant d'être à nouveau classé dans une des trois cases. Pour un gestionnaire de dossiers, avoir un taux d'erreurs important ne signifie pas seulement voir disparaître ses chances de promotions ou d'augmentations, cela signifie également subir rappels à l'ordre et récriminations de la part des cadres du service, qui sont eux-mêmes notés en fonction des résultats de leurs agents.

L'ensemble de ce dispositif de « contrôle-rétributions-sanctions » pousse les uns comme les autres à la productivité et à la perfection, mais génère également une pression constante qui a des conséquences sur le traitement des dossiers. Dans la mesure où seuls les dossiers basculés dans la case « accords » font l'objet d'un contrôle - les dossiers mis dans les cases « refus » ou « retours » ne le sont pas -, nous avons régulièrement observé que pour éviter d'accroître leur taux d'erreur, les gestionnaires de dossiers privilégient les retours ou les refus. Outre que cela réduit les risques de remontrances et d'erreurs, les deux cas profitent directement à leur productivité puisqu'ils sont comptés comme autant de dossiers traités. Les pratiques d'instruction n'étant pas toujours très claires pour les agents, ils ont une certaine marge de manœuvre quant à l'application des règles (Cognet et al., 2009 ; Gabarro, 2008). La liste des documents qui permet de prouver la stabilité de trois mois de résidence d'un étranger en situation irrégulière n'est pas exhaustive. Face à un document non listé, le gestionnaire de dossier peut hésiter à prendre le risque d'augmenter son taux d'erreur en l'acceptant et préférer retourner le dossier pour demander un autre document. Les « retours » et les « refus », qui prennent moins de temps à être traités, se présentent alors comme une solution de facilité et de rapidité pour les agents, bien que les cadres tentent de limiter les « retours » qu'ils perçoivent comme une augmentation de la charge de travail de leur service, les dossiers étant instruits deux fois. En conséquence, les cadres du service AME mettent en place, avec l'accord de leur hiérarchie, des pratiques managériales pour liquider leur « stock » le plus rapidement possible, en favorisant les refus ou, au moins, en accélérant les retours au moyen d'une procédure de vérification rapide de certaines rubriques à l'ouverture du courrier. Depuis juillet 2009, les dossiers dont les rubriques « date d'entrée en France » et « membres de la famille résidant en France » ne sont pas renseignées sont retournés aux demandeurs et ce, même si ces informations ne conditionnent pas l'accès à l'AME. Ici, c'est en fait la marge d'interprétation laissée par les directives nationales quant à l'application des règles qui est invoquée (Cognet et al., 2009). Cette pratique multiplie les « retours », mais génère aussi des retours mal faits : en se focalisant sur ces rubriques, le gestionnaire de dossiers ne vérifie pas si le dossier contient bien les éléments conditionnant l'octroi de l'AME. Et si ce n'est pas le cas, le demandeur se verra imposer un deuxième retour, rallongeant encore les délais.

Enfin, en vue de diminuer le volume de dossiers en attente, les cadres demandent aux agents d'enregistrer minutieusement la date de renvoi du dossier. Les demandeurs ne disposant que de trente jours pour envoyer à la caisse les documents manquants, tout dépassement de date permet des « refus implicites », c'est-à-dire de classer le dossier dans la case « refus » sans même en informer le demandeur. Un mode de gestion, extrêmement rapide, qui accroît de façon conséquente la productivité des employés et celle du service AME. 
La course à la productivité fait assez bien ressortir la marge de manœuvre des caisses en matière d'interprétation des règles et les effets qu'elle peut avoir sur le traitement des dossiers. Les circulaires ministérielles concernant l'AME sont en fait retranscrites deux fois : une première fois par la Caisse nationale qui donne les instructions générales aux CPAM, et une deuxième fois par chaque caisse primaire qui traduit les injonctions de la Caisse nationale en fonction de son dispositif organisationnel propre. C'est à ce niveau-là que des interprétations variables peuvent être faites, se traduisant par des modes d'organisation et des lectures variables du droit qui expliquent les différences de traitement des demandeurs de l'AME dans les CPAM des Champs et des Lys. Or, pour les demandeurs qui se voient refuser leur dossier par erreur ou du fait d'une lecture restrictive du droit, ces refus n'étant pas supervisés, seule la voie des recours reste ouverte. Mais les recours gracieux sont instruits par les caisses qui les étudient selon la même interprétation des textes et dans le même contexte de gestion et de productivité.

Par cette politique des retours de dossiers, les employés du service AME ne barrent pas l'accès aux soins aux demandeurs (sauf quand le « retour » se transforme en « refus implicite »), mais le retardent. Or la plupart des étrangers en situation irrégulière en France, ne se résolvent à faire leur première demande d'AME que quand ils ont un besoin urgent de soins. Les difficultés sur lesquelles ils butent tout au long des procédures que nous venons de décrire, et les délais que celles-ci occasionnent, peuvent avoir de graves conséquences sur leur état de santé. Lorsque les demandeurs sont renvoyés chez eux par les gestionnaires de clientèle, leur passage à l'accueil n'est pas pris en compte : ce n'est qu'une fois le dossier considéré comme complet, qu'il est tamponné et daté. Or c'est à partir de cette date que seront ouverts les droits à l'AME alors que si ce même dossier avait été directement adressé par la poste au service AME, c'est la date de réception du courrier dans le service qui aurait été retenue pour l'ouverture des droits, même si le dossier avait fait des aller-retour entre temps ${ }^{29}$.

\section{CONCLUSION}

Au final, c'est l'ensemble des pratiques et des cadres règlementaires que nous avons analysés qui forment un système aboutissant à "l'écrémage " d'une partie des demandeurs de l'AME. La politique managériale du service AME se combine avec les errances administratives générées par les hésitations ou décisions malencontreuses dans les accueils des caisses, pour produire des refus et des retours en nombre. Les procédures elles-mêmes sont ainsi porteuses de traitements différentiels entre demandeurs. Mais, c'est d'abord la distinction entre l'AME et la CMU qui entraîne de nombreuses difficultés pour les demandeurs de l'AME. Maintenues à la marge du système, devant se rendre dans des locaux spécifiques, dépendant de procédures particulières, ces personnes se retrouvent confrontées à un véritable parcours du combattant. Cette course d'obstacles à l'obtention de l'AME est aggravée par la mise en place de nouvelles normes de management au sein des caisses d'assurance maladie. Cette politique du chiffre se retrouve aujourd'hui, au-delà des entreprises privées comme les centres d'appel (Calderon, 2005) ou McDonald (Weber, 2011), dans de nombreuses administrations du service public telles que les préfec-

29 À condition, que la personne réponde à la demande sous trente jours. 
tures (Spire, 2007), la Poste (Cartier, 2003), les caisses d'allocations familiales (Siblot, 2006) ou les hôpitaux (Belorgey, 2010). Face aux exigences de productivité évaluée à partir d'indicateurs quantitatifs biaisés, les responsables et agents des caisses d'assurance maladie, comme ceux des autres administrations et entreprises soumises aux mêmes impératifs, mettent en place des stratégies pour pouvoir traiter toujours plus de dossiers, toujours plus vite. Leurs marges de manœuvre en matière d'interprétation des textes sont utilisées pour améliorer le rendement des services, diminuer les « stocks » de dossiers en attente et atteindre les objectifs chiffrés. Différentes lectures du droit peuvent ainsi être faites selon les caisses, mais également selon les agents. En effet, comme l'a montré Spire (2005 et 2007), les agents des préfectures interprètent les règles et traitent les demandes de régularisation différemment en fonction, entre autres, de leur place dans la hiérarchie préfectorale, de leur niveau de diplôme ou de leur trajectoire sociale. Ces différences se retrouvent également parmi les agents de l'assurance maladie. L'accès aux soins relève ainsi d'une certaine forme d'arbitraire, les demandeurs n'étant ni traités, ni informés de la même manière selon l'agent qui les reçoit : certains agents, le plus souvent ceux en fin de carrière, prennent le temps de recevoir les demandeurs et de leur expliquer les démarches à suivre, sans mesurer leur temps. D'autres leur donnent seulement le formulaire et le document explicatif. Certains leur réclament l'ensemble des originaux, quitte à les faire revenir, tandis que d'autres se satisfont des photocopies. Les demandeurs se trouvent ainsi pris au milieu de logiques organisationnelles et de productivité qui compliquent toujours plus leurs démarches. Mais ils sont conscients, à force de fréquenter les administrations, de l'existence d'interprétations et de pratiques différentes selon les agents.

Pour finir, il s'avère que toutes les stratégies que nous avons observées dans les services, du fait des soupçons de fraude existants et de la politique de « maîtrise des risques » mise en place par les caisses, s'exercent au détriment des demandeurs de l'AME et de leur santé. Les retours de dossiers, solution plébiscitée dans les caisses, allongent sans cesse la date d'ouverture de droits, retardant d'autant l'accès aux soins. 


\section{Références bibliographiques}

BARON Pierre, BORY Anne, TOURETTE Lucie, CHAUVIN Sébastien et JOUNIN Nicolas (2011) On bosse ici, on reste ici ! La grève des sans-papiers : une aventure inédite, Paris, La Découverte, $312 \mathrm{p}$.

BELORGEY Nicolas (2010) L'hôpital sous pression. Enquête sur le " nouveau management public », Paris, La Découverte, 330 p.

BERNARD Olivier (2011) Santé publique et politique sécuritaire : l'union contre-nature, Humanitaire, [en ligne] consulté le 04/03/2012. URL : http://humanitaire.revues.org/index1108. html

CALDERON Jose Angel (2005) L'implication quotidienne dans un centre d'appels : les nouvelles « initiatives éducatives », Travailler, 1 (13), pp. 75-94.

CARDE Estelle (2009) Quinze ans de réforme de l'accès à une couverture maladie des sans-papiers : de l'Aide sociale aux politiques d'immigration, Mouvements, 3 (59), pp. 143-156.

CARDE Estelle (2007) Les discriminations selon l'origine dans l'accès aux soins, Santé Publique, 19 (2), pp. 99-109.

CARDE Estelle (2006a) Les discriminations selon l'origine dans l'accès aux soins, Étude en France métropolitaine et en Guyane française, Thèse de Doctorat en Santé publique, sous la direction de Didier Fassin, Université Paris XI, 540 p.

CARDE Estelle (2006b) « On ne laisse mourir personne ». Les discriminations dans l'accès aux soins, Travailler, 16 (2), pp. 57-80.

CARTIER Marie (2003) Les facteurs et leurs tournées. Un service public au quotidien, Paris, La Découverte, $329 \mathrm{p}$.

CASTEL Robert (1995) Les métamorphoses de la question sociale, Paris, Gallimard, 812 p.

CATTACIN Sandro and CHIMIENTI Milena (2006) Difference Sensitivity in the Field of Migration and Health. National policies compared, Genève, Research Report of the Department of Sociology of the University of Geneva, $61 \mathrm{p}$.

COGNET Marguerite, GABARRO Céline et ADAM-VÉZINA Émilie (2009) Entre droit aux soins et qualité des soins, Hommes et Migrations, 1282, pp. 54-65.

FASSIN Didier (1997) La santé en souffrance, in Didier Fassin, Alain Morice et Catherine Quiminal Éds., Les Lois de l'inhospitalité. Les politiques de l'immigration à l'épreuve des sans-papiers, Paris, La Découverte, pp. 107-123.

FASSIN Didier, CARDE Estelle, FERRÉ Nathalie et MUSSO DIMITIJEVIC Sandrine (2002) Un traitement inégal. Les discriminations dans l'accès aux soins. Rapport d'étude $n^{\circ} 5$, Bobigny, CRESP.

FASSIN Didier et MORICE Alain (2001) Les épreuves de l'irrégularité : les sans-papiers entre déni d'existence et reconquête d'un statut, in Dominique Schnapper Éd., Exclusions au coeur de la Cité, Paris, Economica, pp. 261-309.

FERRÉ Nathalie (1997) La production de l'irrégularité, in Didier Fassin, Alain Morice et Catherine Quiminal Éds., Les Lois de l'inhospitalité. Les politiques de l'immigration à l'épreuve des sanspapiers, Paris, La Découverte, pp. 47-64.

FROTIÉE Brigitte (2006) La réforme française de la Couverture maladie universelle, entre risques sociaux et assurance maladie, Lien social et Politiques-RIAC, La santé au risque du social, Printemps 2006, pp. 33-44.

FROTIÉE Brigitte (2004) La fabrique du droit social : l'exemple de la CMU. Sous la direction de Jacques Commaille, Rapport au Fonds CMU.

GABARRO Céline (à paraître) Quand la Roumanie adhère à l'Union européenne : quels changements dans l'accès à la Couverture Maladie Universelle pour les ressortissants communautaires ?, in Frédéric Le Marcis et Kàtia Lurbe I Puerto Éds., Exclusion, Reconnaissance et Expériences des " endo-étrangers » en Europe. Rroms, Manouches, Gitans et gens du voyage, Paris, Academia-Le Bruyant. 
GABARRO Céline (2008) Discriminations dans l'accès aux soins : l'influence des présupposés dans le traitement de l'Aide médicale de l'État (AME), mémoire de master en sociologie, sous la direction de Marguerite Cognet, Université Paris 7, $144 \mathrm{p}$.

HACHIMI ALAOUI Myriam et NACU Alexandra (2010) Soigner les étrangers en situation irrégulière : des politiques migratoires aux postures professionnelles, Hommes et migrations, 1284, pp. 163-173.

HUMA-NETWORK (2009) L'accès aux soins des personnes sans papiers et des demandeurs d'asile dans 10 pays de l'Union européenne, Rapport HUMA, Législation et pratique, 186 p., [en ligne]. URL : http://www.huma-network.org/averroes_fr

IZAMBERT Caroline (2010) 30 ans de régression dans l'accès aux soins, Plein droit, 86, octobre, [en ligne]. URL : http://www.gisti.org/spip.php?article2102

MAILLE Didier et TOULLIER Adeline (2009), Les dix ans de la CMU. Un bilan contrasté pour l'accès aux soins des migrants, Hommes et Migrations, 1282, pp. 54-65.

MAILLE Didier, TOULLIER Adeline et VOLOVITCH Pierre (2005) L'aide médicale de l'État : comment un droit se vide de son sens faute d'être réellement universel, Revue de droit sanitaire et social, 4, pp. 543-554.

MARTIN Pascal (2011) Gestion de la file d'attente et invisibilisation des précaires. Mensonge institutionnalisé dans une caisse d'assurance maladie, Actes de la recherche en sciences sociales, 4 (189), pp. 34-41.

MATH Antoine (2009) Droit à la santé des ressortissants communautaires vivant en France. Les difficultés d'accès à la protection maladie et aux soins, Hommes et migrations, 1282, pp. 122-135.

MBAYE Elhadji Mamadou (2009) Sida et immigration thérapeutique en France : mythe ou réalités, Sciences sociales et santé, 27 (1), pp. 43-62.

MÉDECINS DU MONDE (2009) L'accès aux soins : un droit fondamental non respecté en Europe. Deuxième rapport de l'Observatoire Européen de Médecins du Monde, $23 \mathrm{p}$.

MURARD Numa (2001) La protection sociale. Paris, La Découverte, Repères, 128 p.

MIPES (2003) Regards sur la couverture maladie universelle, rapport de la Mission d'Information sur la Pauvreté et l'Exclusion Sociale en Île-de-France, 13 p.

PALIER Bruno (2005) Gouverner la sécurité sociale, Paris, PUF, 502 p.

SIBLOT Yasmine (2006) Faire valoir ses droits au quotidien. Les services publics dans les quartiers populaires, Paris, Presses de Sciences Po, 348 p.

SIMÉANT Johanna (1998) La cause des sans-papiers, Paris, Presses de Sciences Po, 504 p.

SPIRE Alexis (2007) L'asile au guichet. La dépolitisation du droit des étrangers par le travail bureaucratique, Actes de la recherche en sciences sociales, 4 (169), pp. 4-21.

SPIRE Alexis (2005) L'application du droit des étrangers en préfecture, Politix, 1 (69), pp. 11-37.

WEBER Hélène (2011) Du ketchup dans les veines. Pratiques managériales et illusions : le cas McDonald's, Paris, Erès, 220 p. 


\title{
Les demandeurs de l'aide médicale d'État pris entre productivisme et gestion spécifique
}

\author{
Céline Gabarro
}

En 2000 est créée l'aide médicale d'État (AME), une couverture maladie réservée aux personnes en situation irrégulière. Dans cet article, nous nous intéressons aux conséquences de cette spécificité sur l'accès aux soins de ces personnes dans trois champs : le droit, l'organisation des caisses d'assurance maladie et les pratiques des agents de ces caisses (aussi bien les agents d'accueil qui reçoivent les demandeurs, que les agents du service AME qui instruisent les dossiers). La combinaison de ces trois angles d'approche donnera ainsi à voir du parcours effectué par les personnes en situation irrégulière et des embûches rencontrées. Nous verrons que cantonner les personnes en situation irrégulière au sein d'une prestation qui leur est propre crée un système de santé à plusieurs vitesses, favorisant la réception de ce public dans des lieux distincts ou de manières différenciées et limitant son accès aux soins et aux structures. Nous serons particulièrement attentive à l'impact de la gestion productiviste sur l'accueil de ces personnes et le traitement de leur dossier : les rendements imposés aux agents des caisses et leur manque de formation les poussent à réclamer aux demandeurs plus de justificatifs, différant toujours plus leur accès aux soins.

\section{Applicants for Medical Assistance State Caught between Productivism and Specific Management}

\author{
Céline Gabarro
}

Health coverage only for illegal immigrants, the medical aid (AME) was created in 2000. In this paper, we focus on the consequences of this specificity on access to healthcare for these people in three areas: the law, the organization of health insurance funds, agents and practices of these organizations (both reception staff who receive applicants than service agents investigating cases). The combination of these three angles of approach will give to see the journey made by illegal immigrants and pitfalls encountered. We will see that illegal immigrants confined in their own health care system creates a two-speed system, favouring their reception by the public places separated or differentiated ways and limiting their access to care and structures. We will be particularly attentive to the impact of productivist management on the reception of such persons and the processing of their case: the productiveness imposed on agents and as their lack of training lead them to demand more documents to the applicants, always postponing their access to care. 


\section{Los solicitantes de la Ayuda Médica de Estado tomados entre productivismo y gestión específica \\ Céline Gabarro}

En 2000 es creada la ayuda médica, una cobertura médica reservada a las personas en situación irregular. En este artículo, nos interesamos por las consecuencias de esta especificidad sobre el acceso a la atención médica de estas personas en tres ámbitos: el derecho, la organización de las cajas de seguro de enfermedad, y las prácticas de los agentes de estas cajas (tanto los agentes de acogida que reciben a los solicitantes como los agentes del servicio AME que instruyen los expedientes). La combinación de estos tres ángulos de aproximación dará así a ver el trayecto efectuado por las personas en situación irregular y las trampas encontradas. Veremos que acantonar a las personas en situación irregular en el seno de una prestación que les es propia crea un sistema de salud a varias velocidades, favoreciendo la recepción de este público en lugares distintos o de maneras diferenciadas y limitando su acceso a los cuidados y a las estructuras. Estaremos particularmente atenta al impacto de la gestión productivista sobre la acogida de estas personas y el tratamiento de su expediente: los rendimientos impuestos a los agentes de las cajas y su falta de formación les empujan a reclamarles a los solicitantes más justificantes, difiriendo siempre más su acceso a los cuidados. 